\title{
SRGA J124404.1-632232/SRGU J124403.8-632231: New X-ray pulsar discovered in the all-sky survey by the SRG
}

\author{
V. Doroshenko ${ }^{1}$, R. Staubert ${ }^{1}$, C. Maitra ${ }^{2}$, A. Rau ${ }^{2}$, F. Haberl ${ }^{2}$, A. Santangelo ${ }^{1}$, A. Schwope ${ }^{3}$, J. Wilms ${ }^{4}$, \\ D. A. H. Buckley ${ }^{5,6}$, A. Semena ${ }^{7}$, I. Mereminskiy ${ }^{7}$, A. Lutovinov ${ }^{7}$, M. Gromadzki ${ }^{8}$, \\ L. J. Townsend ${ }^{5,9}$, and I. M. Monageng ${ }^{5,6}$ \\ ${ }^{1}$ Institut für Astronomie und Astrophysik, Sand 1, 72076 Tübingen, Germany \\ e-mail: doroshv@astro.uni-tuebingen.de \\ ${ }^{2}$ Max-Planck-Institut für extraterrestrische Physik, Gießenbachstraße 1, 85748 Garching, Germany \\ ${ }^{3}$ Leibniz-Institut für Astrophysik Potsdam (AIP), An der Sternwarte 16, 14482 Potsdam, Germany \\ ${ }^{4}$ Dr. Karl Remeis-Sternwarte and Erlangen Centre for Astroparticle Physics, Friedrich-Alexander-Universität Erlangen-Nürnberg, \\ Sternwartstr. 7, 96049 Bamberg, Germany \\ ${ }^{5}$ South African Astronomical Observatory, PO Box 9, Observatory Rd, Observatory 7935, South Africa \\ ${ }^{6}$ Department of Astronomy, University of Cape Town, Private Bag X3, Rondebosch 7701, South Africa \\ ${ }^{7}$ Space Research Institute (IKI) of Russian Academy of Sciences, Prosoyuznaya ul 84/32, 117997 Moscow, Russian Federation \\ 8 Astronomical Observatory, University of Warsaw, Al. Ujazdowskie 4, 00-478 Warsaw, Poland \\ 9 Southern African Large Telescope, PO Box 9, Observatory Rd, Observatory 7935, South Africa
}

Received 21 April 2021 / Accepted 13 July 2021

\begin{abstract}
Ongoing all-sky surveys by the eROSITA and the Mikhail Pavlinsky ART-XC telescopes on board the Spectrum-RoentgenGamma (SRG) mission have already revealed over a million of X-ray sources. One of them, SRGA J124404.1-632232/ SRGU J124403.8-632231, was detected as a new source in the third (of the planned eight) consecutive X-ray surveys by ART-XC. Based on the properties of the identified optical counterpart, it was classified as a candidate X-ray binary. We report on the follow-up observations of this source with the Nuclear Spectroscopic Telescope Array, the Neil Gehrels Swift Observatory, and the Southern African Large Telescope, which allowed us to unambiguously confirm the initial identification and establish SRGU J124403.8-632231 as a new X-ray pulsar with a spin period of $\sim 538 \mathrm{~s}$ and a Be-star companion, making it one of the first Galactic X-ray pulsars discovered by the SRG.
\end{abstract}

Key words. pulsars: individual: SRGA J124404.1-632 232/SRGU J124403.8-632 231 - stars: neutron - binaries: general

\section{Introduction}

The Spectrum-Roentgen-Gamma (SRG) mission was launched on July 13, 2019, from Baikonur and started the all-sky survey on December 12, 2019. In total, eight complete scans of the celestial sphere of six months each are foreseen to be conducted with each of the two instruments on board the SRG: eROSITA (Merloni et al. 2012; Predehl et al. 2021) and Mikhail Pavlinsky ART-XC (Pavlinsky et al. 2021) which operate in the $0.2-10 \mathrm{keV}$ band and the 4-30 keV energy range respectively.

Both instruments produce standard X-ray event data and therefore not only images, but also spectra and light curves are available for each point on the sky. At the end of the survey, the eROSITA surveys are expected to be a factor 20-40 more sensitive than the ROSAT All-Sky Survey in the same energy band $\left(\sim 2.5 \times 10^{-15} \mathrm{erg} \mathrm{cm}^{-2} \mathrm{~s}^{-1}\right.$ for eROSITA (Predehl et al. 2021) compared to $\sim 10^{-13} \mathrm{erg} \mathrm{cm}^{-2} \mathrm{~s}^{-1}$ (Boller et al. 2016) for ROSAT). Moreover, SRG will also conduct first ever true imaging all-sky surveys above the ROSAT passband.

A large majority of the several millions of new X-ray sources detected in the survey are expected to be extragalactic objects (i.e., active galactic nuclei and galaxy clusters) and ordinary stars
(Merloni et al. 2012; Predehl et al. 2021). However, a significant expansion of the sample of Galactic X-ray binaries can also be anticipated and will help to obtain important constraints on their luminosity function (Lutovinov et al. 2013; Doroshenko et al. 2014). Of particular interest here are the properties of the faint Be X-ray binaries (BeXRBs), which so far have been mostly discovered with all-sky monitors during their strong outbursts. It is quite clear, however, that the population of known BeXRBs is potentially only the tip of an iceberg because objects exhibiting fainter outbursts or no outbursts at all were likely missed due to the low sensitivity of all-sky monitors (Doroshenko et al. 2014). In comparison with available wide-field instruments, both eROSITA and ART-XC are significantly more sensitive and are expected to unveil at least a part of this hidden population. They will thus facilitate advances in our understanding of the origin and evolution of Galactic X-ray binaries.

On 2021 January 27, during the third consecutive allsky survey, the Mikhail Pavlinsky ART-XC telescope on board the SRG observatory detected the bright X-ray source SRGA J124404.1-632232 with a flux of $\sim 10^{-11} \mathrm{erg} \mathrm{cm}^{-2} \mathrm{~s}^{-1}$ in the 4-12 keV energy band (Semena et al. 2021). Inspection of the ART-XC data from the previous two surveys in 2020 (see 
Table 1 for a summary of the observations) revealed no excess at this position, therefore the source appeared to be new. However, it was found that an object was detected by eROSITA in all three surveys. This allowed refining its X-ray position and identifying a tentative optical counterpart of the X-ray source (Semena et al. 2021). Near-infrared and X-ray colors, the X-ray flux, and an estimated X-ray luminosity of $\sim 10^{34} \mathrm{erg} \mathrm{s}^{-1}$ implied by the Gaia distance of $\sim 5.8 \mathrm{kpc}$ (Bailer-Jones et al. 2021) strongly suggested that the object may be a new high-mass X-ray binary (HMXB). The variability of the counterpart, in particular, a weak periodic, $\sim 138 \mathrm{~d}$, optical flux variations revealed by the Optical Gravitational Lensing Experiment (OGLE, Mroz \& Udalski 2021) strongly supported this suggestion as similar variability is commonly observed also in other high-mass X-ray binaries with Be-star counterparts. To confirm this identification, we observed the counterpart with the Southern African Large Telescope (SALT; Buckley et al. 2006), revealing a prominent $\mathrm{H} \alpha$ line in emission (Buckley et al. 2021). This is typical for $\mathrm{Be}$ $\mathrm{X}$-ray binaries and also strongly supports the BeXRB nature of the source. It motivated us to conduct follow-up observations of this X-ray source with Nuclear Spectroscopic Telescope Array (NUSTAR).

We here report in detail on the analysis of the observational data of NuSTAR, SALT, and the SRG survey. The paper is organized as follows. In Sect. 2, we first briefly discuss the observations we used and then discuss the analysis steps in detail that led to the identification of the near-infrared counterpart of the $\mathrm{X}$-ray source. We also report details of the analysis for the followup NuSTAR and SALT observations. We then finally discuss our findings in the context of the properties of other BeXRBs in Sect. 3 and summarize the results in Sect. 4.

\section{Observations and data analysis}

As described above, there are two telescopes on board SRG, eROSITA and ART-XC. They were described in detail in Predehl et al. (2021) and Pavlinsky et al. (2021), respectively. Because the exposures achieved in the survey so far are comparatively low, the data from both instruments were mostly used to obtain a precise position of the X-ray source and measure flux variations between the three consequent surveys, whereas NuSTAR data were used for a detailed spectral and timing analysis. Considering the nondetection of the source by ART-XC in the first two surveys, we only used data from the third survey for this instrument. For eROSITA, data from all three surveys were used.

eROSITA data were processed using the current (c946) eSASS (Brunner et al. 2022) pipeline configuration (used to characterize the software and calibration file version), which was also used to derive the source position and to extract X-ray spectra that were modeled jointly with $N U S T A R$ data to improve the low-energy coverage and constraints on the absorption column. Details on calibration and in-orbit background of eROSITA are reported in Dennerl et al. (2020) and Freyberg et al. (2020), respectively. The data from the three all-sky surveys were analyzed independently, although the spectrum of the source was assumed to be the same in all of them, as described below.

ART-XC data were processed with the analysis software ARTPRODUCTS v0.9 with the calibration files version 20200401. A description of the ART-XC telescope and the analysis software can be found in Pavlinsky et al. (2021). Because the source is faint and the effective exposure for ART-XC is significantly shorter as a result of its smaller field of view, it was only possible to detect the source and estimate the number of counts
Table 1. Summary of observations of the source.

\begin{tabular}{llll}
\hline \hline Instrument & $T_{\text {start }}$, MJD & $T_{\text {stop }}$, MJD & Exposure $(k s)$ \\
\hline eROSITA & 58883.36 & 58885.86 & 0.501 \\
& 59068.27 & 59070.43 & 0.380 \\
& 59239.92 & 59241.59 & 0.289 \\
ART-XC & 59240.59 & 59241.42 & 0.172 \\
NuSTAR & 59244.48 & 59245.53 & 54 \\
Swift/XRT & 59248.17 & 59248.77 & 1.7 \\
SALT & 59243.98 & 59244.01 & 2 \\
\hline
\end{tabular}

above the background. We therefore used ART-XC data mostly to improve constraints on the source flux by eROSITA in the third survey. In practice, we attributed all counts detected by ART-XC to two broad energy bins covering the $4-12 \mathrm{keV}$ band and modeled the ART-XC data using the current response. Here we note also that even though ART-XC and eROSITA observed the source at about the same time, the difference in field of view of the two instruments and the scanning pattern of the all-sky survey imply that the observations are not strictly simultaneous. The source flux measured by ART-XC was therefore estimated independently.

In addition to SRG data, a deep (55 ks, obsid. 80660301002) observation of the source with NUSTAR (Harrison et al. 2013) was obtained to characterize the broadband spectrum of the source and search for possible pulsations. The data for this observation were reduced using HEASOFT v6.28 and caldb version 20210210, as described below. Soon after this, the Neil Gehrels Swift Observatory (Swift) also observed the object with the XRT telescope (obsid. 00041978002), so that we used this observation as well.

Furthermore, as part of a transient identification and characterization program being undertaken at SALT (Buckley 2015), we observed the optical counterpart on 2021 January 29 using the Robert Stobie Spectrograph (RSS) in low-resolution mode $(R=420)$. In particular, two spectra with $1 \mathrm{ks}$ exposure each covering the region 3300-9000 $\AA$ were obtained starting 23:34:05 UTC. The summary of all observations is presented in Table 1.

Finally, to study the properties of the optical counterpart, we used the archival OGLE I-band light curve published by Mroz \& Udalski (2021). It is available through the OGLE XROM service $^{1}$.

\subsection{X-ray position and near-infrared counterpart}

To determine the X-ray position of the source, we considered the rectified source positions from three eROSITA surveys as independent measurements and combined them following the procedure described in Doroshenko et al. (2020). This allowed us to fix the final X-ray position as $\alpha_{\mathrm{J} 2000.0}=12^{\mathrm{h}} 44^{\mathrm{m}} 03.57, \delta_{\mathrm{J} 2000.0}=-$ $63^{\circ} 22^{\prime} 28^{\prime \prime}$. 1 with an uncertainty of about $1^{\prime \prime} .4$ at $1 \sigma$ or $\sim 3^{\prime \prime} .7$ at a $3 \sigma$ confidence level (not accounting for potential systematic uncertainties (up to $5^{\prime \prime}$ ) of individual eROSITA measurements). Fig. 1 shows that the estimated X-ray position coincides with the near-infrared source 2MASS J12440380-6322320, which was thus suggested as the tentative optical counterpart of the X-ray source (Semena et al. 2021). Considering the variability reported by Mroz \& Udalski (2021), we observed the object with SALT (Buckley et al. 2021) to finally confirm this identification.

1 http://ogle.astrouw. edu.pl/ogle4/xrom/srga_j 124404 . 1-632232.html 

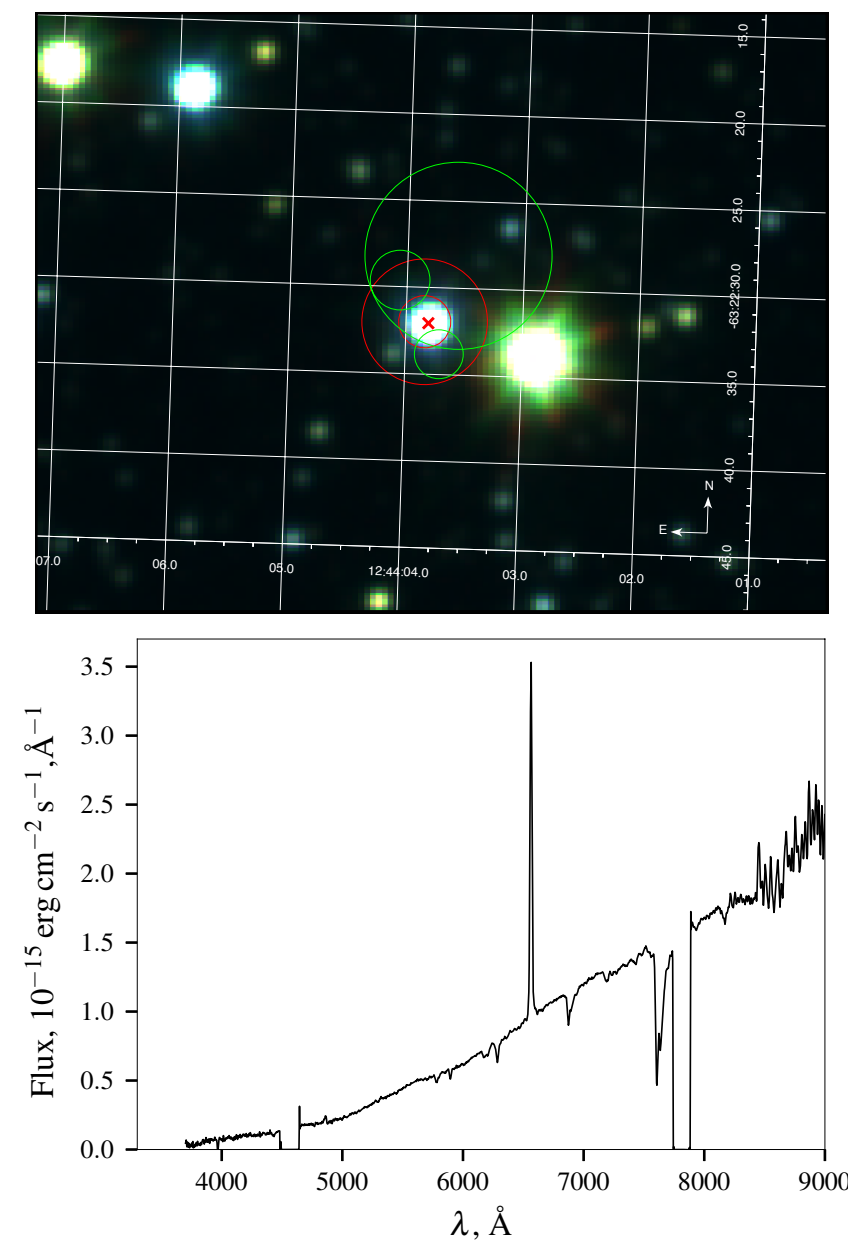

Fig. 1. Top panel: VVV $\left(J H K_{S}\right)$ image of the region around the $\mathrm{X}$-ray source. The green circles indicate the source position as observed by eROSITA in eRASS1-3, the red contours represent the $1 \sigma$ and $3 \sigma$ uncertainties for the refined position obtained as described in the text. The 2MASS position of the suggested near-infrared counterpart is indicated by the red cross. Bottom panel: spectrum of the optical counterpart as observed by SALT, note the prominent $\mathrm{H} \alpha$ line.

In particular, the proposed optical counterpart was observed using the low-resolution $3001 \mathrm{~mm}^{-1}$ transmission grating that is typically used for the spectral classification of transients. The RSS spectra were reduced using the PYSALT primary data reduction pipeline (Crawford et al. 2012), which corrects for bias, gain, and detector mosaic amplifier cross-talk. We then used $\mathrm{IRAF}^{2}$ for the remaining data reduction steps, namely wavelength calibration, background subtraction extraction of the 1D spectra, and relative flux calibration (the design of SALT prevents an absolute flux calibration).

The combined SALT spectrum presented in Fig. 1 shows a strong $\mathrm{H} \alpha$ emission line, a weaker $\mathrm{H} \beta$ emission line, and a largely featureless red continuum that rises at longer wavelengths. It is thus consistent with a highly reddened early-type Be star located in the Galactic plane at $\sim 6 \mathrm{kpc}$ (Semena et al. 2021) and strongly suggests that the X-ray source is indeed likely a Be X-ray binary (Buckley et al. 2021).

The $\mathrm{H} \alpha$ line is single peaked, but shows a slight asymmetry, with the peak flux on the red side of the line center. The equivalent width (EW) and full width at half maximum (FWHM)

\footnotetext{
2 Image Reduction and Analysis Facility: https:// iraf-community.github.io/
}

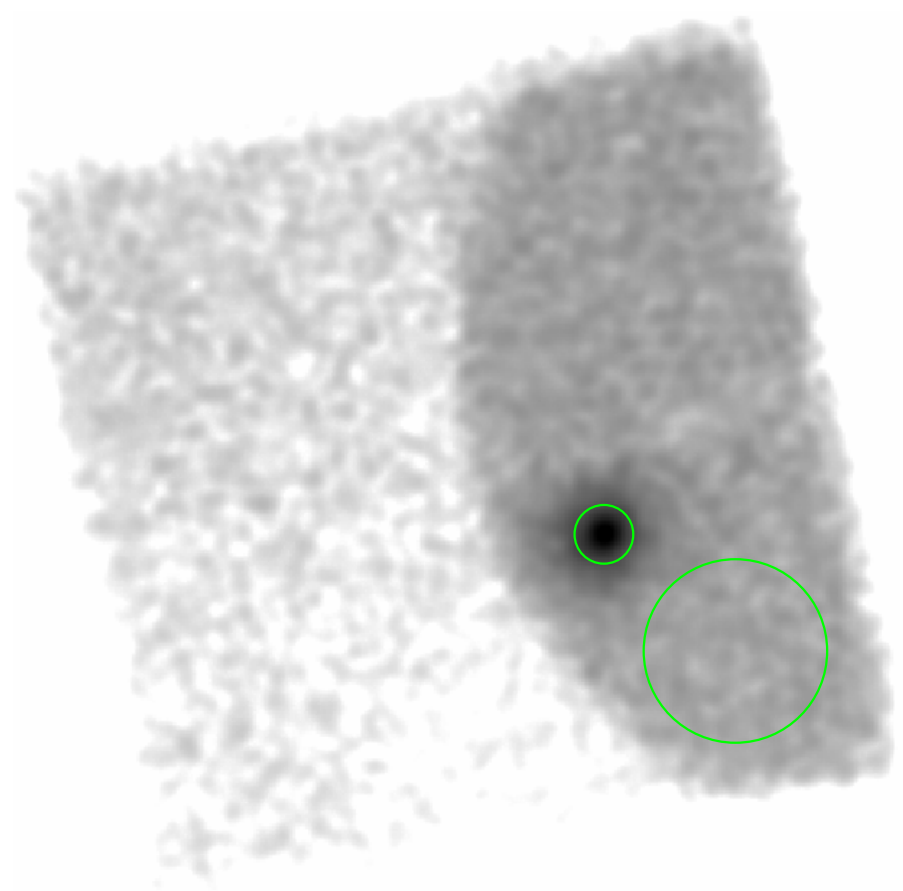

Fig. 2. Smoothed plane image for the NUSTAR FPMA detector unit in the $3-20 \mathrm{keV}$ energy band. Note the stray light contamination from GX 301-2. The locations of the source and background extraction regions are also shown (green circles).

of the line are measured as $-54.2 \pm 2.7 \AA$ and $17.55 \pm 0.48 \AA$, respectively. The $\mathrm{H} \beta$ line $\mathrm{EW}$ is approximately $-5 \AA$, although this is hard to measure precisely because at the blue end of the spectrum, the signal-to-noise ratio $(\mathrm{S} / \mathrm{N})$ is lower. The $\mathrm{H} \alpha \mathrm{EW}$ is larger than that of most other BeXRBs. Based on the proposed orbital period of 138 days (Mroz \& Udalski 2021) and the well-known correlation between orbital period and $\mathrm{H} \alpha \mathrm{EW}$ in BeXRBs (Reig 2011), the expected EW would be about $-35 \AA$, therefore our measurement suggests that the disk has grown to be larger than expected for this orbital period. The system can therefore indeed can be expected to be active in X-rays.

\subsection{NUSTAR data analysis}

We here mainly focus on the NUSTAR observation of the source that was obtained as part of the approved guest observer program targeting X-ray binaries discovered by the SRG. The observation was triggered following the SALT observation and was specifically optimized to mitigate potential stray-light contamination from the bright nearby X-ray pulsar GX 301-2. In particular, it was scheduled to occur outside of the regular pre-periastron flares observed from this X-ray binary (Doroshenko et al. 2010).

As illustrated in Fig. 2, it was unfortunately not possible to completely avoid contamination. This resulted in a low S/N for SRGU J124403.8-632231, particularly in the hard band above $20 \mathrm{keV}$. For the spectral and timing analysis in the hard band, an optimized extraction region of $32^{\prime \prime}$ was therefore chosen to achieve the optimal $\mathrm{S} / \mathrm{N}$ in the $20-80 \mathrm{keV}$ energy band, as described by Vybornov et al. (2018). However, taking into account that most of the photons are detected below $20 \mathrm{keV}$, a larger region $\left(72^{\prime \prime}\right)$ was selected for the timing analysis in the soft band, using the same procedure. Because of the apparent stray-light contamination from GX 301-2, the background was also extracted from a contaminated area on the same chip away 


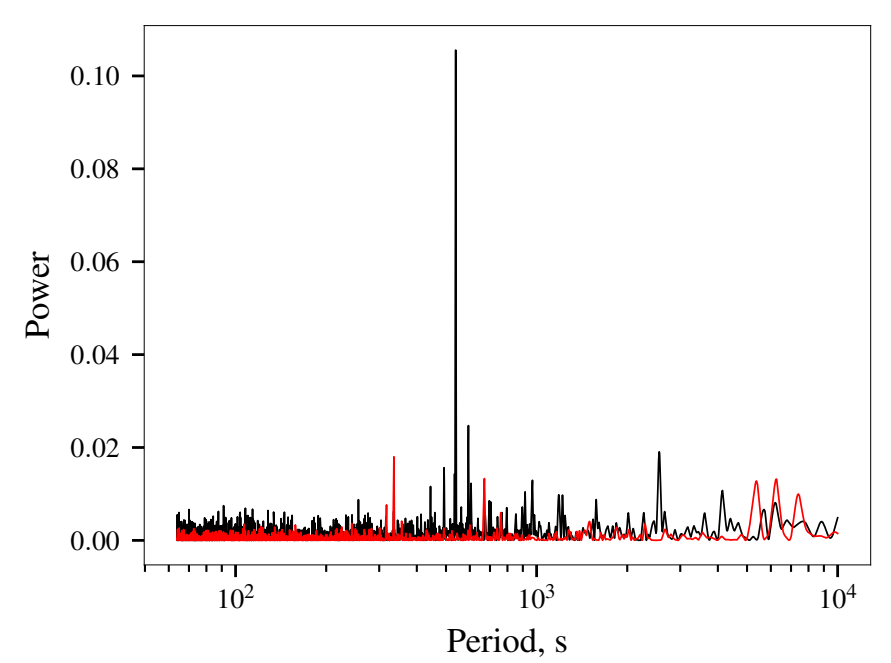

Fig. 3. Lomb-Scargle periodogram for source (black) and background (red) light curves. The $\sim 670 \mathrm{~s}$ and $\sim 335 \mathrm{~s}$ peaks from GX 301-2, which are visible in the background light curve, are not present in the source light curve and do not overlap the pulsed signal from SRGA J124404.1-632232/SRGU J124403.8-632231.

from the source using a circular region with a radius of $100^{\prime \prime}$. We verified that the choice of the location of the background region (as long as it is extracted from a stray-light contaminated region) does not affect any of our conclusions.

Timing analysis. To search for possible pulsations, we extracted a background-subtracted light curve in the $3-20 \mathrm{keV}$ energy band with a time resolution of $16 \mathrm{~s}$, combining the events extracted from the two NUSTAR units. We applied barycentric correction and then searched for pulsations using the generalized Lomb-Scargle periodogram (Zechmeister \& Kürster 2009). The result is presented in Fig. 3, where a strong peak corresponding to a period of $\sim 538.56 \mathrm{~s}$ is obvious (the false-detection probability as defined in Zechmeister \& Kürster (2009) is $\log P$ $-87)$.

We note that this peak is not associated with the pulsations from the nearby GX 301-2, which has a longer period of $\sim 670 \mathrm{~s}$ that is clearly detected in the periodogram of the background light curve, but not for the backgroundsubtracted light curve. The detected pulsations imply that the $\mathrm{X}$-ray source is an accreting neutron star. We therefore conclude that SRGA J124404.1-632232/SRGU J124403.8-632231 is likely indeed a new Be X-ray pulsar.

In order to refine the period value and in order to estimate its uncertainty, we used the phase-connection technique (Deeter et al. 1981). This approach allowed us to determine the final pulse period value as $P=538.70(5) \mathrm{s}$. This value does not account for Doppler delays associated with the orbital motion of the pulsar. The reason is that the orbital parameters of the system are not yet known and cannot be estimated using the comparatively short NUSTAR observation (with respect to the expected orbital period).

To investigate the dependence of the pulse profiles of the source on energy, we extracted light curves in the 3-5, 5-10, 10-20, and 20-40 keV energy bands and folded them with the determined period using 32 equally spaced phase bins. Again, the light curves from the two NUSTAR units were coadded and background was subtracted. Figure 4 shows that the pulse profile is single peaked, sinusoidal, and exhibits no strong variations with energy. The pulsed fraction, defined here as $\max (r)-$

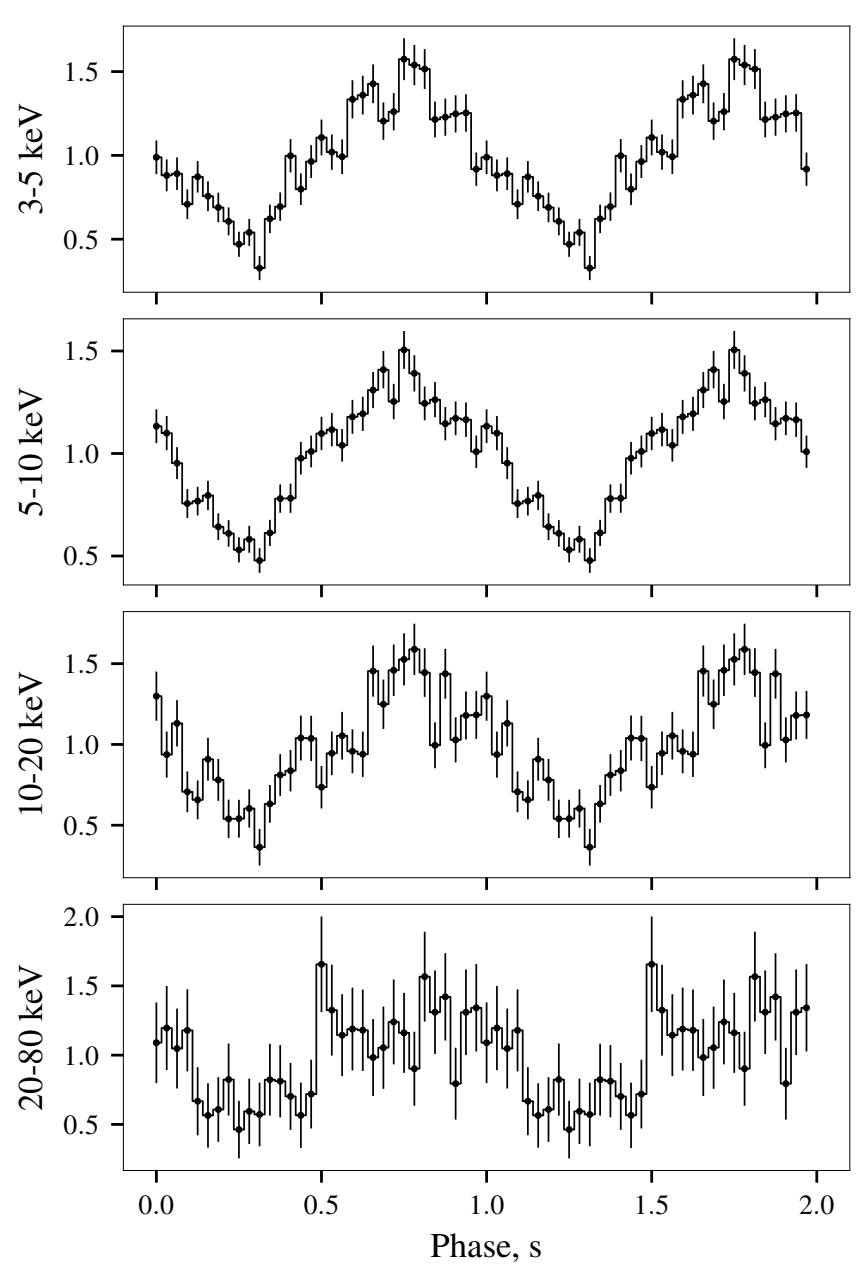

Fig. 4. Normalized background-subtracted pulse profiles of SRGA J124404.1-632232/SRGU J124403.8-632231 as a function of energy as observed by NUSTAR.

$\min (r) / \max (r)+\min (r)$, where $r$ is the count rate, is high at all energies at $\sim 55-90 \%$, and as illustrated in Fig. 5, it does not exhibit the clear increasing trend that is typical for X-ray pulsars (Lutovinov \& Tsygankov 2009) or other features, except for a slight drop in the $5-10 \mathrm{keV}$ band that is likely related to the chosen binning of the pulse profile and comparatively large measurement uncertainties.

Spectral analysis. Taking into account that the stray-light contamination is mostly relevant in the hard band, we extracted the broadband NUSTAR spectrum using the region that was optimized for the hard band, that is, using the extraction radius of $32^{\prime \prime}$. The SRG spectra were extracted as described above. The Swift/XRT spectrum of the source was extracted using the online tools provided by $\operatorname{UKSSDC}^{3}$ (Evans et al. 2009). Considering the low statistics in all spectra with the exception of NUSTAR, we modeled six data sets (i.e., eROSITA spectra extracted using the srctool task separately for the three surveys, the ART-XC spectrum for the third survey, the NuSTAR spectra extracted separately from the two units, and the spectrum from the Swift/XRT pointing carried out soon after NuSTAR) using the same model, including a cross-normalization constant to account for differences in flux. As described, this includes

3 https://www.swift.ac.uk/user_objects/index.php 


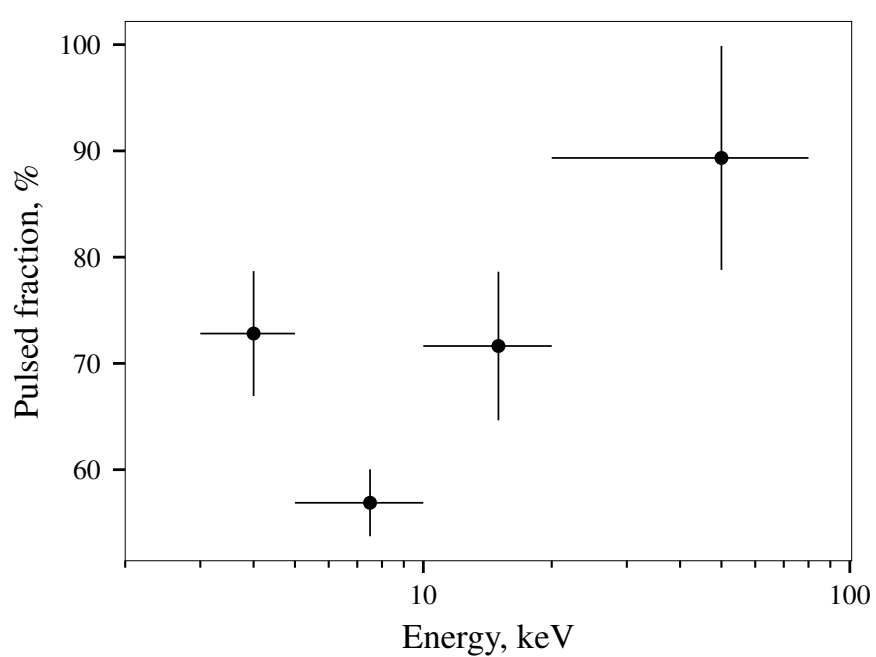

Fig. 5. Pulsed fraction of SRGA J124404.1-632232/SRGU J124403.8632231 as a function of energy as observed by NuSTAR.

contemporary observations by eROSITA and ART-XC, which are not strictly simultaneous because of the different field of view. This causes different scan lengths over the source for the two instruments. All spectra were grouped to contain at least one count per energy bin and were modeled using Xspec v12.11.1 using the Cash (Cash 1979) and Anderson-Darling (Anderson \& Darling 1952) as fit and test statistics. This also necessitated the use of Monte Carlo sampling for the final estimates of parameter uncertainties and the goodness of fit (using the chain and goodness commands in Xspec).

We considered several phenomenological models that are typically used to describe the spectra of X-ray pulsars to model the broadband spectrum of SRGA J124404.1-632232/ SRGU J124403.8-632231. In particular, we first attempted to model the spectrum with absorbed cutoff power-law models, that is, cutoffpl and highecut models multiplied by a crossnormalization constant to account for differences in the absolute flux calibration of individual instruments and flux variations between observations and TBabs (Wilms et al. 2000) to account for absorption. However, the cutoff energy was not constrained effectively in either case, reducing both models to a simple power law. This is unexpected for an X-ray pulsar. Nevertheless, the best-fit parameters for the absorbed power-law model are listed in Table 2 and presented in Fig. 6. We note that the goodness of fit assessed using a set of spectra simulated based on the best-fit power-law model using the goodness command was not acceptable (more than $95 \%$ of the simulations yielded lower statistics than the data). Other models therefore needed to be considered.

We note that the estimated broadband unabsorbed source flux for the power-law model of $\sim 2.3 \times 10^{-11} \mathrm{erg} \mathrm{cm}^{-2} \mathrm{~s}^{-1}$ implies a luminosity of $\sim 10^{35} \mathrm{erg} \mathrm{s}^{-1}$ for the assumed distance of $5.8 \mathrm{kpc}$ (Bailer-Jones et al. 2021), that is, SRGA J124404.1-632232/ SRGU J124403.8-632231 appears to be more similar to lowluminosity pulsars such as X Persei than to classical Be transients. We accordingly also considered a model consisting of two Comptonization components represented by the CompTT model (Titarchuk 1994) with linked seed temperatures used to describe spectra of $\mathrm{X}$ Persei and other similar sources (Doroshenko et al. 2012, 2021; Tsygankov et al. 2019c,b,a; Mushtukov et al. 2021; Lutovinov et al. 2021). The continuum was again modified to account for interstellar absorption using a TBabs component multiplied by a cross-normalization constant to account for flux variations and differences in absolute
Table 2. Broadband spectral modeling results for SRGA J124404.1-632232/SRGU J124403.8-632231 using absorbed power law (powerlaw) and two-component Comptonization models (CompTT2).

\begin{tabular}{lll}
\hline \hline & powerlaw & CompTT2 \\
\hline$C_{\mathrm{FMPB}}$ & $1.08(3)$ & $1.08(4)$ \\
$C_{\mathrm{XRT}}$ & $0.6(1)$ & $0.6(1)$ \\
$C_{\mathrm{e} 1}$ & $0.6(1)$ & $0.6(1)$ \\
$C_{\mathrm{e} 2}$ & $0.16(9)$ & $0.17(4)$ \\
$C_{\mathrm{e} 3}$ & $1.3(4)$ & $1.3(3)$ \\
$C_{\mathrm{ART}}$ & $3.3(7)$ & $2.9(3)$ \\
$N_{\mathrm{H}}, 10^{22} \mathrm{~cm}^{-2}$ & $4.1(7)$ & $2.6(3)$ \\
$\Gamma, \mathrm{keV}$ & $2.11(4)$ & \\
$k T_{0}, \mathrm{keV}$ & & $0.12(3)$ \\
$k T_{1} / k T_{2}, \mathrm{keV}$ & & $1.85(8) / 8.3(8)$ \\
$\tau_{1} / \tau_{2}$ & & $20(1) / 13(1)$ \\
$A_{\Gamma / 1 / 2} / 10^{-3}$ & $2.6(3)$ & $1.6(2) / 0.08(1)$ \\
$F_{\mathrm{obs} / \mathrm{src}}, 10^{-11} \mathrm{erg} \mathrm{cm}^{-2} \mathrm{~s}^{-1}$ & $1.14 / 2.26$ & $1.0 / 1.3$ \\
\hline $\log P_{\mathrm{AD}} / \mathrm{dof}$ & $-8.81 / 1432$ & $-9.89 / 1427$ \\
$\mathrm{BIC}$ & 46 & 64 \\
\hline
\end{tabular}

Notes. Test statistics probability $\left(\log P_{\mathrm{AD}}\right)$ and $\mathrm{BIC}$ values are quoted.

flux calibration. The best-fit results are presented in Table 2. The fit is not only statistically preferred to the power-law model, but more importantly, it is also statistically acceptable (verified using the goodness command). To compare the two models, we used the Bayesian information criterion (BIC, see, i.e., Kass \& Raftery 1995), which indeed indicates a very strong preference for the Comptonization model (see Table 2 for BIC values). The best-fit parameters are also similar to those observed in other X-ray pulsars observed in a low-luminosity state (see, e.g., the papers cited above). We therefore conclude that SRGA J124404.1-632232/SRGU J124403.8-632231 indeed shows a two-component spectrum similar to that of other $\mathrm{X}$-ray pulsars observed at comparable luminosities, as described in detail in Sect. 3.

Finally, the comparison of spectrum normalization obtained by various instruments also allowed us to assess the variability of the source on long timescales. As illustrated in Table 2, the source flux appears to be variable by a factor of six between the observations. We emphasize, however, that because the duration of the individual scans is shorter than the spin period of the source, and high pulsed fraction revealed by NUSTAR, most of this variability is likely associated with the short-term variability over the pulse period rather with than long-term flux changes. We therefore conclude that the long-term flux of the source remains comparatively stable, without the clear outbursts that are typical for the BeXRBs that were detected so far.

\section{Discussion}

As discussed above, the observed properties of the newly discovered BeXRB pulsar SRGU J124403.8-632231 are rather similar to those of other long-period pulsars observed at low luminosities. In particular, similar spectra were reported from the transient XRBs 1A 0535+262 (Tsygankov et al. 2019b) and GX 304-1 (Tsygankov et al. 2019c) when observed in quiescence. Another object for which a similar X-ray spectrum was observed is the only known persistent BeXRB, X Persei, which 


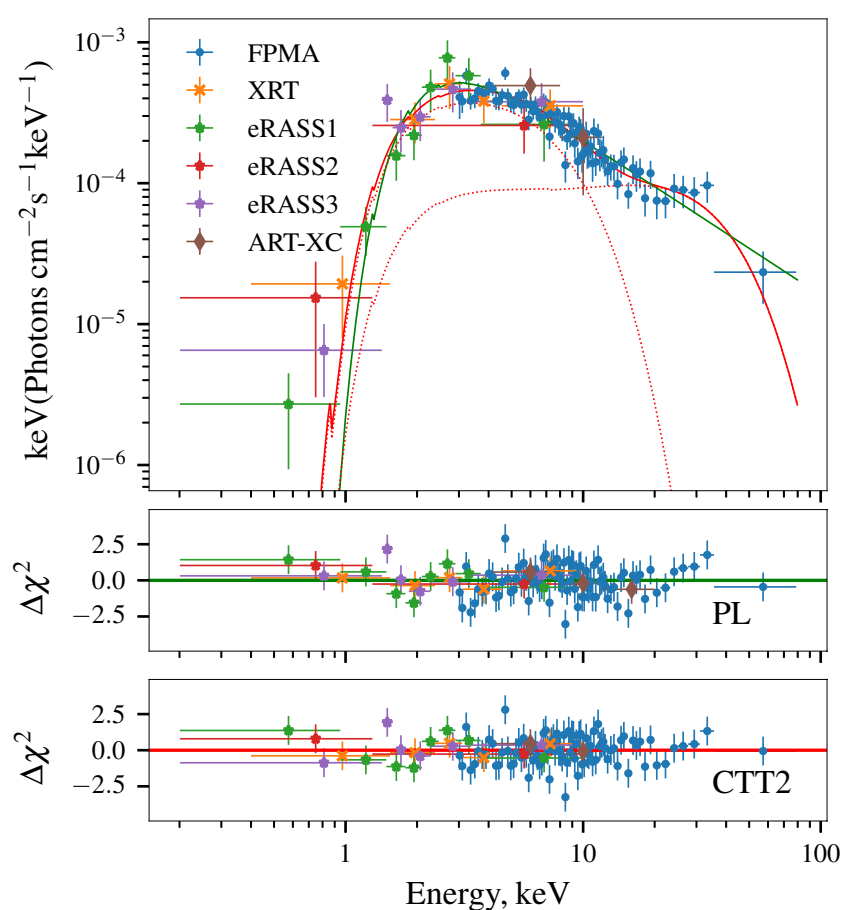

Fig. 6. Unfolded spectrum of the source assuming the best-fit CompTT2 model. All spectra were rebinned (to 10 (eROSITA and Swift/XRT) or 25 counts (NuSTAR) per energy bin) and scaled using the best-fit crossnormalization constants listed in Table 2. For clarity, only FPMA data for NUSTAR are shown because the spectra from the two modules look nearly identical.

historically was only observed in the low-luminosity state, presumably due to the longer orbital period and wider separation between the neutron star and the donor.

SRGU J124403.8-632231 appears to be similar to this source, that is, it appears to be a low-luminosity persistent pulsar. The relatively long orbital period of $\sim 138 \mathrm{~d}$ suggested by Mroz \& Udalski (2021) and the nondetection of strong outbursts, despite the observed prominent $\mathrm{H} \alpha$ emission line indicating an extended decretion disk around the primary (Reig et al. 2016), suggests that the pulsar is probably indeed far from the companion and thus unlikely to cross the circumstellar disk to undergo strong outbursts. For long-period pulsars the accretion is expected to proceed from the cold nonionized accretion disk that is regularly replenished by the wind of the primary (Tsygankov et al. 2017). As a result, a relatively stable luminosity of $\sim 10^{34-35} \mathrm{erg} \mathrm{cm}^{-2} \mathrm{~s}^{-1}$, consistent with the observations, might be expected to be maintained for a long time.

We note, however, that the orbital period of the source is not yet reliably known, so that it cannot be fully excluded that the object will eventually undergo an outburst. The suggestion of the orbital period by Mroz \& Udalski (2021) is based on the detection of several short episodes in which the companion brightened by $\sim 0.1 \mathrm{mag}$ (illustrated in Fig. 7). However, considering that only five such episodes can be identified in the light curve, we cannot be entirely sure that they are periodic and associated with orbital motion. A search for periodicity in the raw OGLE light curve reveals several marginally significant peaks, and the peak at $\sim 140 \mathrm{~d}$, that is, slightly longer than the period suggested by Mroz \& Udalski (2021), is only one of them. In particular, the longer period of $\sim 225 \mathrm{~d}$ also appears to be consistent with most of the brightening episodes (see, e.g., Fig. 7). Furthermore, the $225 \mathrm{~d}$ period also fits the correlation between $\mathrm{H} \alpha \mathrm{EW}$ and orbital
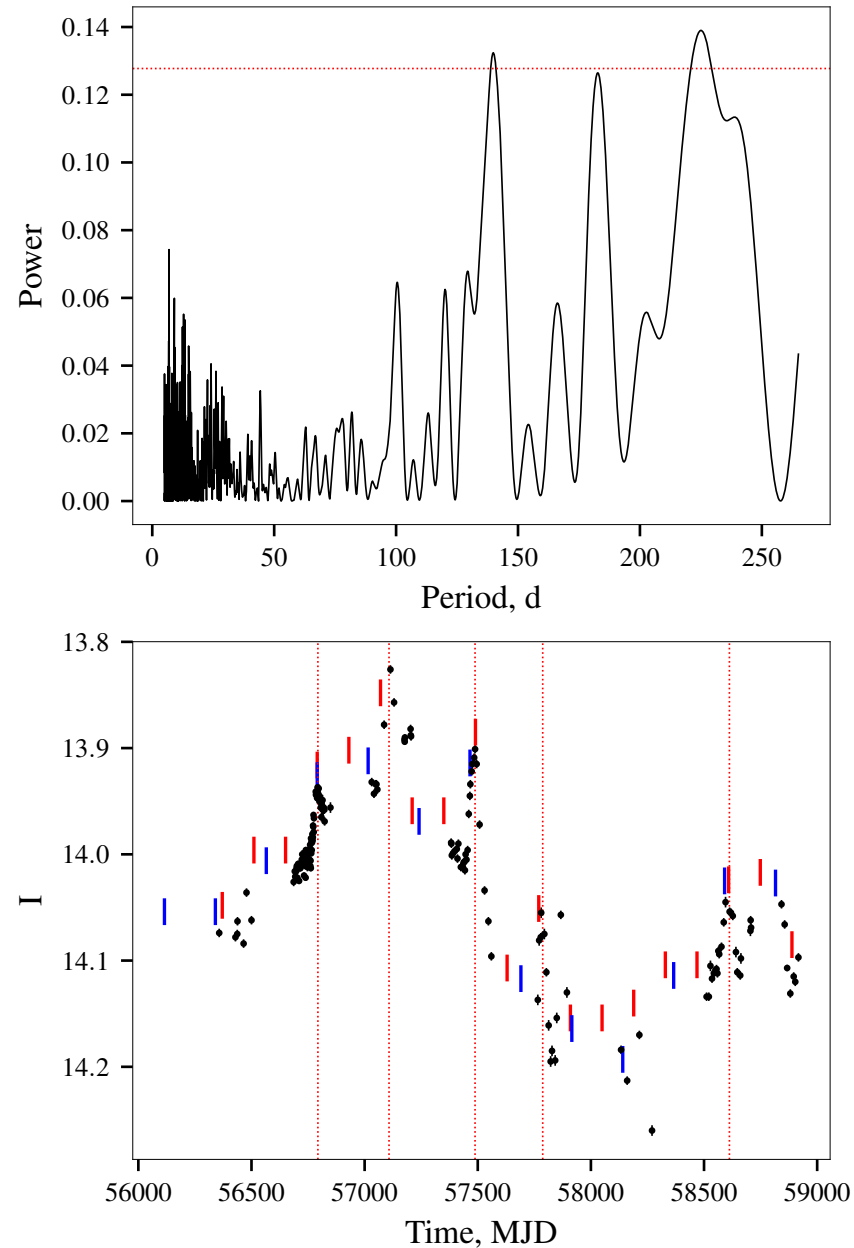

Fig. 7. Generalized Lomb-Scargle periodogram for OGLE $I$-band light curve of SRGA J124404.1-632232/SRGU J124403.8-632231 counterpart (top). The red line indicates the expected $3 \sigma$ upper limit on the noise amplitude. Two peaks exceeding the noise level correspond to periods of $\sim 138$ and $\sim 225$ d. OGLE I-band light curve (bottom, black points). The five short, quasi-periodic flares are indicated with dotted red lines. The short vertical lines indicate expected flare timings assuming that they are related to the orbital phase, with an orbital period of either $138 \mathrm{~d}$ (red) or $225 \mathrm{~d}$ (blue).

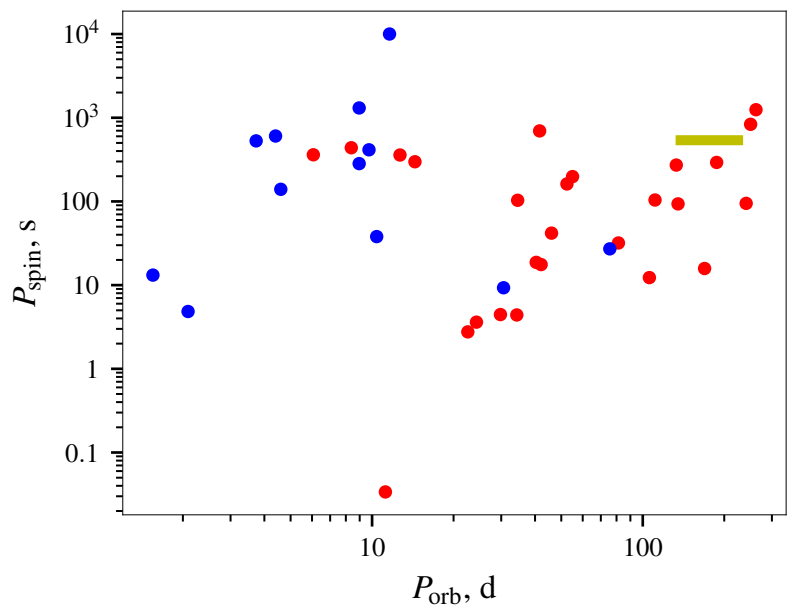

Fig. 8. Orbital period - spin period (Corbet 1986) diagram for windaccreting (blue) and Be (red) high-mass X-ray binaries. The position of SRGA J124404.1-632232/SRGU J124403.8-632231 is indicated with the yellow line assuming an orbital period from 138 to $225 \mathrm{~d}$. 
period better (discussed in Sect. 2.1). This period corresponds to the expected EW of $\sim 50 \AA$ (Reig 2011), consistent with our measurement with SALT. The two suggested values are also consistent with expectations based on the relation between spin and orbital periods for X-ray pulsars (Corbet 1986), as illustrated in Fig. 8 (here shown based on the data reported in the catalog of the Galactic HMXBs by Liu et al. 2006). Further monitoring of the source in X-ray and optical bands is therefore required to clarify the long-term behavior of the source.

\section{Conclusions}

SRGA J124404.1-632232/SRGU J124403.8-632231 is one of the first new Galactic X-ray pulsars identified in the all-sky Xray survey of the SRG (see also Lutovinov et al. 2022), and it is the first confirmed Galactic Be X-ray binary (several such objects have also been detected in the Large Magellanic Cloud, where longer exposures are available; see, e.g., Maitra et al. 2020, 2021b,a; Haberl et al. 2020). It is not yet fully clear, however, whether the source is a persistent low-luminous pulsar similar to X Persei or a low duty cycle transient Be X-ray binary. The nondetection of a strong outburst with a peak luminosity of $\sim 10^{36-37} \mathrm{erg} \mathrm{s}^{-1}$ typical for other BeXRBs, despite the observed prominent $\mathrm{H} \alpha$ emission line from the companion, which indicates the presence of an expanded decretion disk (Reig et al. 2016) around the donor, suggests, however, that the former is more likely. We note that pre-launch estimates indicate that several tens of objects like this one are expected to be detected in the eROSITA survey (Doroshenko et al. 2014), and SRGA J124404.1-632232/SRGU J124403.8-632231 might just be the first of them. Only the SRG can unveil the population of these faint persistent objects. We are searching for more of them in the survey.

Acknowledgements. This work is based on data from eROSITA, the primary instrument aboard SRG, a joint Russian-German science mission supported by the Russian Space Agency (Roskosmos), in the interests of the Russian Academy of Sciences represented by its Space Research Institute (IKI), and the Deutsches Zentrum für Luft- und Raumfahrt (DLR). The SRG spacecraft was built by Lavochkin Association (NPOL) and its subcontractors, and is operated by NPOL with support from the Space Research Institute (IKI) and Max Planck Institute for Extraterrestrial Physics (MPE). The development and construction of the eROSITA X-ray instrument was led by MPE, with contributions from the Dr. Karl Remeis Observatory Bamberg and ECAP (FAU Erlangen-Nuernberg), the University of Hamburg Observatory, the Leibniz Institute for Astrophysics Potsdam (AIP), and the Institute for Astronomy and Astrophysics of the University of Tübingen, with the support of DLR and the Max Planck Society. The Argelander Institute for Astronomy of the University of Bonn and the Ludwig Maximilians Universität Munich also participated in the science preparation for eROSITA The eROSITA data shown here were processed using the eSASS/NRTA software system developed by the German eROSITA consortium. The Mikhail Pavlinsky ART-XC telescope is the hard X-ray instrument on board the SRG observatory, a flagship astrophysical project of the Russian Federal Space Program realized by the Russian Space Agency, in the interests of the Russian Academy of Sciences. ART-XC was developed by the Space Research Institute (IKI, Moscow) and the Russian Federal Nuclear Center - All-Russian Scientific Research Institute for Experimental Physics (RFNC-VNIIEF, Sarov) with the participation of the NASA's Marshall Space Flight Center (MSFC). The ART-XC team thanks the Russian Space Agency, Russian Academy of Sciences and State Corporation Rosatom for the support of the SRG project and ART-XC telescope. We thank the NUSTAR SOC for their invaluable help in the quick scheduling of the observations. This research has made use of data, software and/or web tools obtained from the High Energy Astrophysics Science Archive Research Center (HEASARC), a service of the Astrophysics Science Division at NASA/GSFC and of the Smithsonian Astrophysical Observatory's High Energy Astrophysics Division. This work made use of data supplied by the UK Swift Science Data
Centre at the University of Leicester. A.S., I.M. and A.L. acknowledge support from Russian Science Foundation via grant 19-12-00423. The SALT observations reported here were triggered by SRG and obtained through the SALT Large Science program (2018-2-LSP-001; PI: Buckley). Polish participation in SALT is funded by grant no. MNiSW DIR/WK/2016/07. D.A.H.B. is supported by the National Research Foundation (NRF) of South Africa. M.G. is supported by the EU Horizon 2020 research and innovation programme under grant agreement no. 101004719.

\section{References}

Anderson, T. W., \& Darling, D. A. 1952, Ann. Math. Stat., 23, 193

Bailer-Jones, C. A. L., Rybizki, J., Fouesneau, M., Demleitner, M., \& Andrae, R. 2021, AJ, 161, 147

Boller, T., Freyberg, M. J., Trümper, J., et al. 2016, A\&A, 588, A103

Brunner, H., Liu, T., Lamer, G., et al. 2022, A\&A, 661, A1 (eROSITA EDR SI) Buckley, D. A. H. 2015, in Proceeding of Science, SALT Science Conference 2015 (SSC2015; https://pos.sissa.it/250/021/pdf), 1

Buckley, D. A. H., Swart, G. P., \& Meiring, J. G. 2006, Proc. SPIE, 6267, 62670Z Buckley, D. A. H., Gromadzki, M., Townsend, L., et al. 2021, ATel, 14364, 1

Cash, W. 1979, ApJ, 228, 939

Corbet, R. H. D. 1986, MNRAS, 220, 1047

Crawford, S. M., Still, M., Schellart, P., et al. 2012, Astrophysics Source Code Library [record ascl: 1207.010]

Deeter, J. E., Boynton, P. E., \& Pravdo, S. H. 1981, ApJ, 247, 1003

Dennerl, K., Andritschke, R., Bräuninger, H., et al. 2020, SPIE Conf. Ser., 11444, 114444Q

Doroshenko, V., Santangelo, A., Suleimanov, V., et al. 2010, A\&A, 515, A10

Doroshenko, V., Santangelo, A., Kreykenbohm, I., \& Doroshenko, R. 2012, A\&A, 540, A1

Doroshenko, V., Ducci, L., Santangelo, A., \& Sasaki, M. 2014, A\&A, 567, A7

Doroshenko, V., Suleimanov, V., Tsygankov, S., et al. 2020, A\&A, 643, A62

Doroshenko, V., Santangelo, A., Tsygankov, S., \& Ji, L. 2021, A\&A 647, A165

Evans, P. A., Beardmore, A. P., Page, K. L., et al. 2009, MNRAS, 397, 1177

Freyberg, M., Perinati, E., Pacaud, F., et al. 2020, SPIE Conf. Ser., 11444, 1144410

Haberl, F., Maitra, C., Carpano, S., et al. 2020, ATel, 13609, 1

Harrison, F. A., Craig, W. W., Christensen, F. E., et al. 2013, ApJ, 770, 103

Kass, R. E., \& Raftery, A. E. 1995, J. Am. Stat. Assoc., 90, 773

Liu, Q. Z., van Paradijs, J., \& van den Heuvel, E. P. J. 2006, A\&A, 455, 1165

Lutovinov, A. A., \& Tsygankov, S. S. 2009, Astron. Lett., 35, 433

Lutovinov, A. A., Revnivtsev, M. G., Tsygankov, S. S., \& Krivonos, R. A. 2013, MNRAS, 431, 327

Lutovinov, A., Tsygankov, S., Molkov, S., et al. 2021, ApJ, 912, 17

Lutovinov, A. A., Tsygankov, S. S., Mereminskiy, I. A., et al. 2022, A\&A, 661, A28 (eROSITA EDR SI)

Maitra, C., Haberl, F., Carpano, S., et al. 2020, ATel, 13610, 1

Maitra, C., Haberl, F., Maggi, P., et al. 2021a, MNRAS, 504, 326

Maitra, C., Haberl, F., Vasilopoulos, G., et al. 2021b, A\&A, 647, A8

Merloni, A., Predehl, P., Becker, W., et al. 2012, ArXiv e-prints [arXiv:1209.3114]

Mroz, P., \& Udalski, A. 2021, ATel, 14361, 1

Mushtukov, A. A., Suleimanov, V. F., Tsygankov, S. S., \& Portegies Zwart, S. 2021, MNRAS, 503, 5193

Pavlinsky, M., Tkachenko, A., Levin, V., et al. 2021, A\&A, 650, A42

Predehl, P., Andritschke, R., Arefiev, V., et al. 2021, A\&A, 647, A1

Reig, P. 2011, Ap\&SS, 332, 1

Reig, P., Nersesian, A., Zezas, A., Gkouvelis, L., \& Coe, M. J. 2016, A\&A, 590, A122

Semena, A., Doroshenko, V., Arefiev, A., et al. 2021, ATel, 14357, 1

Titarchuk, L. 1994, ApJ, 434, 570

Tsygankov, S. S., Mushtukov, A. A., Suleimanov, V. F., et al. 2017, A\&A, 608, A17

Tsygankov, S. S., Doroshenko, V., Mushtukov, A. A., Lutovinov, A. A., \& Poutanen, J. 2019a, A\&A, 621, A134

Tsygankov, S. S., Doroshenko, V., Mushtukov, A. A., et al. 2019b, MNRAS, 487, L30

Tsygankov, S. S., Rouco Escorial, A., Suleimanov, V. F., et al. 2019c, MNRAS, 483, L144

Vybornov, V., Doroshenko, V., Staubert, R., \& Santangelo, A. 2018, A\&A, 610, A88

Wilms, J., Allen, A., \& McCray, R. 2000, ApJ, 542, 914

Zechmeister, M., \& Kürster, M. 2009, A\&A, 496, 577 